\title{
How an educator characterizes scientific domains and disciplinary relationships: A case of change
}

\author{
Deborah Hemingway ${ }^{1}$, Vashti Sawtelle ${ }^{2,3}$, and Chandra Turpen ${ }^{4}$ \\ ${ }^{1}$ University of Maryland, College Park, Biophysics Program, College Park, MD 20724 USA \\ ${ }^{2}$ Michigan State University, Lyman Briggs College, East Lansing, MI 48824 USA \\ ${ }^{3}$ Michigan State University, Department of Physics, East Lansing, MI 48824 USA \\ ${ }^{4}$ University of Maryland, College Park, Department of Physics, College Park, MD 20724 USA
}

\begin{abstract}
In this project, we seek to understand how instructors scaffold and support students' interdisciplinary engagement in an Introductory Physics for the Life Sciences (IPLS) course developed at the University of Maryland, College Park (UMCP) as part of a broader design research project. Here we analyze how one educator positioned scientific disciplines in relation to one another over a two-year time span. Our analysis of the educator's discourse in the classroom demonstrates shifts in the messages the educator sends about the domains of physics and biology. In year one, physics and biology were largely described as distinct and different, but in year two they were more frequently described as similar, complementary, and overlapping.
\end{abstract}

PACS: 01.40.Fk, 01.40.gb, 01.30.Cc

\section{INTRODUCTION}

The reformed Introductory Physics Course for the Life Sciences (IPLS) at the University of Maryland, College Park (UMD) is the physics component of the National Experiment in Undergraduate Science Education (NEXUSPhysics) project, a response to calls from the medical and biological science communities for a reformed undergraduate curriculum. [1,2] Other research initiatives on the NEXUS-Physics project have contributed accounts of what students are getting out of these experiences but left underspecified what instructors in these environments are communicating regarding the nature of given disciplines and what such communications may do to enable or constrain students' learning opportunities. [3,4] An examination of how faculty position the disciplines relative to one another can help us understand how this influences students' ways of building connections between disciplines.

In this work, we examine two sequential implementations of the NEXUS-physics course to discern how the educator shifted in the presented relationship between the disciplines. When lecturing in front of a classroom, educators may find themselves making statements without realizing the implications of such statements. The commentary can thus shift drastically without detection from the educator that such is occurring. We, as educators, communicate many of our expectations for students, only some of which are deliberate and intentional. [5] This work analyzes the effects of such a shift with hopes of raising awareness of this phenomenon within the Physics Education Research (PER) community.

\section{METHODS}

The initial implementations of the course were recorded by three separate video cameras - two on student groups and one focused on the educator. This work analyzes the instructor view recordings of the first 15 out of 45 lectures of the first semester of the course across two different implementations. We coded these 15 lectures with eleven separate codes ranging from discussion on the nature of biology, to discussions on the use of multiple representations. On average, coded discussions are approximately 30-60 seconds in length but range from six seconds to over eight minutes. This work focuses on the coding for discussions regarding two codes: nature of physics (NOP), and nature of biology (NOB).

For reliability checks, an independent reviewer viewed and coded data from six arbitrarily selected distinct lectures to serve as a comparison. The results from this reviewer were approximately identical with all eleven codes matching, and start and end times matching within 30 seconds. Our codes and commentary were also compared with commentary created by members of the research team made while present during the initial presentation of the lectures. The interpretations and commentary were also discussed on a continual basis with researchers from the UMD PER group for confirmation purposes.

\section{RESULTS FROM FIRST COURSE ITERATION}

In the first iteration of the course, our results demonstrate that the educator positions the disciplines of physics and biology as being separate and unique. The disciplinary differences highlighted include variation in the mathematical preparedness of distinct groups of students, the derivation of knowledge in the disciplines, and differences in the ways the disciplines think. In the first course iteration, there are five instances in which NOB and NOP appear together. In four of these five discussions, the educator highlights clear differences between the 
disciplines. One instance contains a mixed message by first discussing a similarity between biology and physics then transitioning to discussing differences. We now describe the substance of the differences that the educator describes.

\section{A. Differences in Mathematical Preparedness}

In the first iteration, the educator establishes as salient the students' mathematical preparation through statements indicating that this course was originally targeted to a specific type of student - the student who is defined as being "less well prepared in mathematics". The following discourse occurred during a discussion regarding NOB.

Educator: So here is the reason why we are doing what we are doing. Over the past decade there has been a lot of calls to modernize the undergraduate education for biology and pre-medical students... Calls for a multidisciplinary, competency-based science education both for biology and pre med students that is going to be less constrained and restricted than the previous environment.... But the point of this course... was a course that was created originally for a whole crew of different kinds of students but mostly students who were less well prepared in mathematics. And what they did is they took the engineering course, and they cut it back, and they shortened it up.

Many of the students enrolled in this course are aspiring health professionals majoring in biological sciences. In this segment, these students are grouped together as not well prepared in mathematics and are in contrast to the group of those that would be taking the engineering course.

\section{B. Differences in Derivation of Knowledge}

In the first iteration of the course, biology is positioned as a discipline where one should be able to summon knowledge that has been learned from authority. In contrast physics is positioned as a discipline in which one should not memorize facts, but one in which students should be able to generate knowledge. As one example, the students are expected to be familiar with the anatomy of mammalian and fish respiratory systems and to recall on demand during a course discussion:

Eugene: My answer was sort of like hers. I said that instead of being in the outside, it started developing those ridges on the inside so there is a larger volume, you could pack all of those ridges in the same surface area.

Educator: And what might you call that structure that you are building?

Eugene: In fish, they're lungs. In mammals, we call those the bronchi of our lungs.

Educator: Fish don't have lungs.

Eugene: Oh I mean gills. $\cdots$

Educator: Right. Yeah. Great. So you're supposed to have had biology before you take this course so I'm gonna feel free to toss in questions like that. And since I'm not a biologist, ...you'll have to keep me honest if I go astray on the biology. Okay? Because you probably know a lot more biology than I do.

When a student makes an error in his recall of the anatomy, the error is quickly challenged by the educator, then rectified by the student. The educator then states that the students are "supposed to have had biology" implying that the educator will expect recall of biological facts. The course educator then further distances himself by claiming that he is "not a biologist", thereby excusing himself from the requirement to recall biological facts, as those labeled "biologists" are asked to do. This separation of expectations further distances the disciplines from each other.

As the discussion in this day of class continues, the way that physicists approach problems is positioned as different from the way that biologists approach problems:

Educator: And he [the speaker at a physics colloquium] basically explained that with a... very simple model, throwing away most of the biology, just focusing. So this is the way physicists do biology, and this is the way physicists do physics.

This example demonstrates an "us-versus-them" mentality by describing physicists as disregarding the complexity of the biological system being examined.

\section{Differences in Thinking}

During a lecture mid-way through the semester, the educator led an explicit discussion highlighting the differences between biology and physics, comments associate thinking and reasoning with physics and memorizing with biology. The educator opens this discussion by describing a conference on teaching physics for biology students in which he was encouraged to teach "them" how to think like "us". The telling of this story communicates a barrier between the disciplines. The educator comments that they have focused on this, the difference, for thirty years.

The educator then states explicitly that there is a gap, meaning that the different disciplines are separate and unique. The educator sets up two separate columns on the board, further separating the disciplines as distinct. The remaining activity involves placing generalizations about the nature of each discipline in the respective column. There is only one comment, "breaks into pieces" that is later placed in the middle as representing both "thinking like a physicist" and "thinking like a biologist". All other comments are placed in one column or the other (see Table 1 for what is written on the board). 
Educator: I'm interested in what's your perception at this point. So what do you think is different about what I do from what you've seen in your biology classes? What do we do differently in physics? (pause) And there's no right answer to this, right? I am asking about your perception.

Jasper: I don't know. I'm kind of having trouble seeing a difference. I guess I just kind of think of it as like they're sciences.

Educator: OK. You think it's similar.

Sameer: I mean, I'm not exactly sure how to explain it but I'm seeing that physicists approach problems differently than biologists do.

Educator: And can you try to be specific in some way? Meaning?

Sameer: Meaning... I don't really know how... I don't know how a physicist does... but it seems that the way you go about doing a problem does feel different.

Educator: Does feel different. OK.

When the educator requests student input, he does so by asking only for the differences. By not asking for similarities, the educator highlights the distinctions and positions the disciplines further apart. Even though the first student to speak states that he is "having trouble seeing a difference," the educator listens to the student but does not provide additional comment nor does he ask the student to elaborate. This acknowledges the student but does not give credit to his stance. In contrast, the next student who offers a difference is asked to elaborate.

The educator continues to listen to students' general comments about the differences until a student describes biology as being more qualitative and positions chemistry closer to physics as working with "a lot of mathematical modeling and quantitative data". The discussion continues with students making statements highlighting differences in physics and biology. The students make comments and the educator writes terms in either column (see Table 1), with the exception of "break into pieces" which is the first and only comment placed into the middle of the two columns.

TABLE 1. Contents from Columns on Blackboard That Were Written By The Professor During A Class Discussion \begin{tabular}{l|l}
\hline Thinking like a physicist & Thinking like a biologist \\
\hline
\end{tabular}

\begin{tabular}{l|l}
\hline Simple models & More complicated models \\
\hline Just do it & Think about big picture \\
\hline More quantitative... & More qualitative \\
\hline $\begin{array}{l}\text { Connect models to basic } \\
\text { principles }\end{array}$ & $\begin{array}{l}\text { Connect models to complex } \\
\text { data }\end{array}$ \\
\hline $\begin{array}{l}\text { Idealized models } \\
\text { Clean principles }\end{array}$ & (No counter listed) \\
\hline Memorize equations \& apply & Memorize concepts...
\end{tabular}

Memorize equations \& apply Memorize concepts...

Think and reason w. equations(No counter listed)
The discussion ends with the addition of the final comment under the "Thinking like a physicist" column stating "Think and reason with equations". No accompanying comment is added to the "Thinking like a biologist" column.

\section{RESULTS FROM SECOND COURSE ITERATION}

A thorough examination of the second iteration of the course reveals a shift in how the nature of physics and nature of biology are described. During the second iteration, the NOP and NOB were described as being more similar than different, and less silo-ed. Similarities within NOB and NOP are noted through discussions regarding their changing nature, use of mathematics, and focus on understanding physical systems. An extended class discussion on the differences between the natures of the disciplines is not present in this iteration. In the second year, there are seven instances in which NOB and NOP appear together. Six of these seven highlight clear similarities between the natures of the two disciplines. One contains a mixed message as it begins by highlighting a generalized difference but then contains a counter example of a similarity. This section highlights the types of similarities the educator discusses.

\section{Similarities in Temporal Change}

On the first lecture of the semester, the educator interjects into his lecture regarding how physics is changing, an example of how biology has changed as well.

Educator: Biology and medicine- everything has changed. When I was in high school, they didn't tell me the right number of chromosomes for human beings. You'd think that they could count that... all of this changes. So there are changes that are happening fast.

The disciplines are aligned with each other in that both are changing disciplines in which novel discoveries are being made, but while they are more aligned, we do still see elements of the "biology as facts" positioning that was present in the first iteration. During a subsequent lecture, the educator again highlights the similarities:

Educator: Equations like this where the quantity is related to the rate of change in the quantity just occurs in science all over the place. And you'll see it in biology. You'll see it in medicine. You'll see it in physics.

In this instance, the educator points out that the examination of an object's rate of change occurs across the disciplines. The educator lists biology, medicine, and physics as all being similar in this regard. 


\section{E. Similarities in Being a Part of Science}

During a discussion on NOB, the educator makes statements on the use of mathematics in the sciences and implicitly includes biology in the category of a science alongside of physics.

Educator: Math is extremely important in science. And this is why they make you [biological science students] take math classes... there's lots of things that is really important about the science that is added on top of the math that makes it much more challenging than the way the math is done in the math classes.

The educator communicates that they take math classes so that it can be used in the sciences. The educator in this message sets up why this class will be relying on the mathematics that the students have already learned.

\section{F. Similarities in Examining Physical Systems}

Embedded in a discussion on the importance of using math in physics, the educator describes both physics and biology in the context of working with a physical system.

Educator: A critical point about the whole process of using math-and it is not just math because once we talk about this you will be able to see that this is very much the same as a lot of what you do in qualitative biologyis that we are going to begin with some physical system. And it could be a biological system. All systems are physical systems.

The educator explicitly brings in biology by commenting on the similarity about both examining physical systems. This shows an aligning of physics and biology in that biologists work with physical systems just as physicists do.

\section{WHAT CONTRIBUTES TO THE EDUCATOR SHIFT IN MESSAGING?}

While we cannot say with certainty what contributed to the educator shifting the messaging about NOP and NOB in the classroom, we can point to a couple of potential influences. During the first iteration of the course, a research team was actively recording students in class and interviewing them outside of class. In between the first and second semester of the first iteration of the course, the research team had a meeting with the educator in which they showed him examples of how his messaging about the disciplines was being taken up by students (e.g. interview transcripts.). Upon reflection of this data the educator noted that some of the ways the students were positioning the disciplines were not how he intended. Additionally, in the second iteration of the course, a biophysics colleague joined

[1] E.F. Redish and D. Hammer, AJP, 77, 629-642 (2009)

[2] E.F. Redish, et al, AJP, 82, 368-377 (2014)

[3] B.D. Geller, et al, AJP, 82, 394-402 (2014) the instructional team. This colleague viewed biology and physics and much more congruent than the original educator, and they had many discussions on how physics and biology were similar. It is likely that both of these types of discussions-with both the research team and the biophysics colleague-contributed to the shift in the educator's messaging from one iteration to the next.

\section{CONCLUSION}

Our analysis shows that the first iteration of the course included comments that positioned the nature of physics as distinct and different from the nature of biology (4 out of 5 instances). The second iteration revealed communications describing the nature of physics and the nature of biology as similar and less siloed (6 out of 7 instances). During the first iteration of the course, the educator led a lengthy eightminute discussion on the differences between "thinking like a physicist" and "thinking like a biologist". This discussion is notably absent from the second iteration of the course.

In follow-up reflections by the educator, he was unaware of any change in his messaging about disciplinary similarities and difference from the first and second iterations of the course. It was only after reviewing this analysis that the educator acknowledged a shift in the communicated nature of the given disciplines. For the PER community, these results demonstrate that without being aware, educators can shift in communicated messages. We, as educators, may believe that we undergo perhaps only small internal shifts that are undetectable externally. Even in the presence of a recognized internal shift, we may not acknowledge any shift in the external messages we communicate. The messages sent to students are sometimes hidden, not only from the students, but also from ourselves.

Further study regarding the change in the discussions on the nature of the given disciplines could examine the effect of these communicated statements on students. Such analysis may contribute to raising instructors' critical awareness of the disciplinary messages they communicate.

\section{ACKNOWLEDGEMENTS}

We would like to acknowledge Kim Moore for her data review and initial efforts in launching this project, as well as other members of the University of Maryland Physics Education Research Group for their feedback regarding this project. In particular, we acknowledge Wolfgang Losert and Joe Redish for their guidance and mentorship; Alice Olmstead and Erin Sohr for their analytical review; and Ben Geller, Ben Dreyfus, and Julia Gouvea for their assistance in data collection and field note creation.

[4] B.W. Dreyfus, V. Sawtelle, C. Turpen, J. Gouvea, and E.F. Redish, APS, PRST - PER. 10, 010115 (2014)

[5] L. Cuban Theory Into Practice Vol. 34, Iss. 1, (1995) 